Hon Syn Chong, MBBS,

Mohd Razif Mohammed Yunus, MS (ORL-HNS),

Norafidaah Ali, MS (Anatomy Pathology)2

'Department of Otorhinolaryngology, Universiti Kebangsaan Malaysia Medical Centre,

Kuala Lumpur, Malaysia

${ }^{2}$ Department of Pathology, Queen Elizabeth Hospital, Sabah, Malaysia
Correspondence: Dr. Mohd Razif bin Mohamad Yunus,

Department of Otorhinolaryngology, UKM Medical Centre

Jalan Yaacob Latif, Bandar Tun Razak, 56000

Cheras, Kuala Lumpur

Malaysia.

Phone: +6019321 2198

Telefax: +60391456675

E-mail: razif72@gmail.com

The authors declared that this represents original material that is not being considered for publication or has not been published or accepted for publication elsewhere, in full or in part, in print or electronic media; that the manuscript has been read and approved by all the authors, that the requirements for authorship have been met by each author, and that each author believes that the manuscript represents honest work.

Disclosures: The authors signed disclosures that there are no financial or other (including personal) relationships, intellectual passion, political or religious beliefs, and institutional affiliations that might lead to a conflict of interest.

\section{An Incidental Nasopharyngeal Carcinoma Coexistent With Primary Nasopharyngeal Tuberculosis}

\author{
ABSTRACT \\ nasopharyngeal tuberculosis and review the literature.

\section{Methods:} \\ Design: Case Report \\ Setting: Tertiary Public University Hospital \\ Patient: One
}

Objective: To describe a case of nasopharyngeal carcinoma coexistent with primary

Results: AA 28-year-old man presented with recurrent sore throat and neck pain with clinically enlarged tonsils. He underwent a routine adenotonsilectomy. Histopathologic examination revealed non-keratinizing squamous cell carcinoma with caseating granulomatous inflammation typical for tuberculosis in the same adenoid specimen. Nasopharyngeal carcinoma was staged T2bN2M0. He was treated with concurrent chemoradiotherapy and a 9-month course of anti tuberculosis treatment. He recovered and remained symptom free one year after treatment.

Conclusion: Nasopharyngeal carcinoma (NPC) and tuberculosis (TB) are both very common diseases in Sabah, East Malaysia. However, it is very rare that both diseases present at the same time and same anatomical area in a patient. Diagnosis can be very challenging and confusing. Multidisciplinary consultations are warranted for appropriate treatment. Combined antituberculosis treatment and concurrent chemoradiotherapy may be appropriate and effective.

Keywords: nasopharyngeal carcinoma, primary nasopharyngeal tuberculosis, adenotonsillectomy

\section{CASE REPORT}

A 28-year-old Malay man presented with the complaint of recurrent sore throat and fever over the past few years. He denied epitaxis, rhinorrhoea, tinnitus, cough, loss of appetite or weight. Clinical examination showed enlarged tonsils. He underwent a routine adenotonsilectomy. Histopathologic examination of the same slide of the adenoid specimen showed fragments of lymphoid tissue covered by respiratory epithelium. The underlying stroma showed infiltrations by clusters and sheets of malignant squamoid cells. There was also presence of caseating necrosis surrounded by epitheloid granulomas with occasional Langhan's giant cells seen. No acid-fast 




bacilli (AFB) were detected. The diagnosis was a non-keratinizing differentiated squamous cell carcinoma with caseating granulomatous inflammation.

He developed a tender left level II cervical lymph node $\left(3 \times 2 \mathrm{~cm}^{2}\right)$ several weeks after the adenotonsillectomy. Fine needle aspiration cytology revealed lymphoid cells with very occasional clusters of atypical cells suspicious for metastasis. No granuloma or AFB was seen.

Tuberculosis work-up results showed three negative serial sputum AFB ZN stains, a normal chest $X$ ray and negative Mantoux test $(0 \mathrm{~mm}$ after 3 days). Hepatitis $B$ and $C$ and HIV screening were non-reactive. Other basic blood investigations were normal. CT scan of the neck revealed a large heterogeneously-enhancing lesion in the nasopharynx causing obliteration of the parapharyngeal space more on left side and left level II cervical lymphadenopathy less than $6 \mathrm{~cm}$. Ultrasound of the abdomen was normal.

He was referred to the infectious disease and oncology teams and thereafter agreed to undergo concurrent chemoradiotherpy (35 fractions of radiotherapy + weekly IV cisplatin $50 \mathrm{mg} / \mathrm{m}^{2}$ followed by three cycles of adjuvant chemotherapy IV $5 F U 700 \mathrm{mg} / \mathrm{m}^{2}+$ IV cisplatin $100 \mathrm{mg} / \mathrm{m}^{2}$ ) and anti TB (EHRZ) treatment for nine months.

Upon completion of his concurrent chemoradiotherapy and anti TB treatment, the left cervical lymph nodes regressed and became unpalpable. He was clinically well on follow up at one year, with no local recurrence visualized on nasopharyngeal endoscopy.
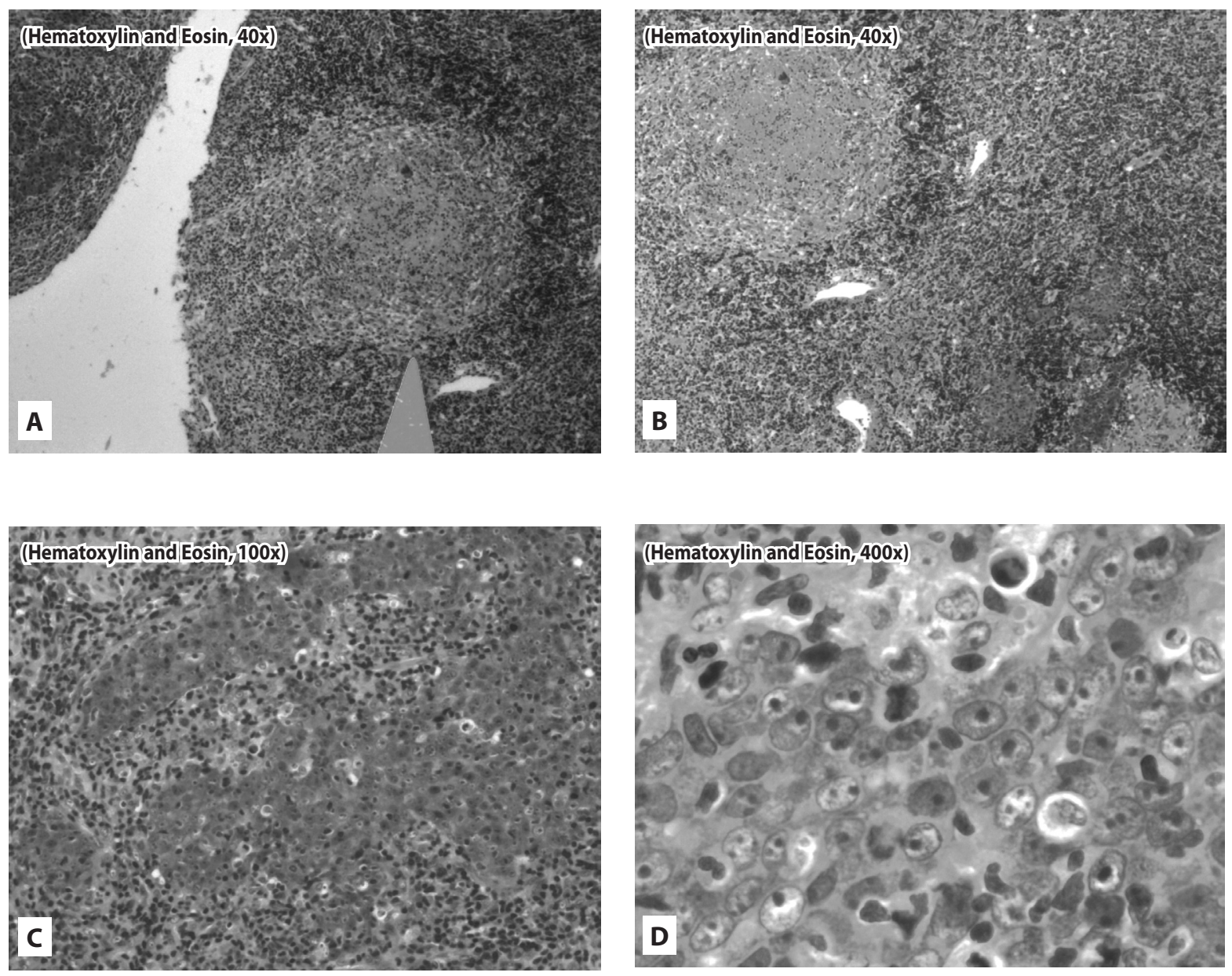

Figure 1 A, B show caseating granuloma with concurrent malignant squamoid cells. (40x) Figure 1 C (100x) and D (400x) show higher magnifications of malignant cells with high mitotic activities. 


\section{DISCUSSION}

Both nasopharyngeal carcinoma (NPC) and tuberculosis are common diseases in this region. However, the incidence of primary nasopharyngeal tuberculosis (NPTB) is very low, comprising about $0.1 \%$ of all tuberculosis cases.' It is even rarer to have a patient present with both pathologies at the same time and in the same anatomical area.

It is a diagnostic dilemma to make a diagnosis as such (NPC with NPTB). It is even more challenging to treat a patient with AFB ZN staining negative in a TB endemic country like Malaysia. In 2007, the state of Sabah contributed slightly more than 3000 of 16,129 new and relapse cases reported in the country. ${ }^{2}$ The incidence of notification of smear-positive PTB is 47 per 100,000 population in Malaysia. ${ }^{3}$

A literature search using Ovid and Medline was done to assess the prevalence of NPC with NPTB and the management experience of others. Concomitant NPC with NPTB has previously been reported by Zalesska-Krecicka et al. ${ }^{4}$ ( 1 case), Wang et al. ${ }^{5}(6$ cases) and Chan et al. ${ }^{6}$ (4 cases). However, all reported a difficult diagnosis and suggested detailed clinical examinations, careful and thorough specimen histopathological examination with necessary staining with more advanced investigations such as Polymerase Chain Reactivity (PCR) and Magnetic Resonance Imaging (MRI). These meticulous steps may help in making a more definitive diagnosis and treatment. Misdiagnosis may lead to NPC diagnosed as NPTB or vice-versa.

As reported by Wang et al. ${ }^{5}$ the clinical manifestations of NPTB include being young; small, numerous, bilateral, soft and movable cervical lymph nodes; concomitant lung tuberculosis; unusual symptoms in the ear and nose; no cranial nerve complications; the nasopharyngeal roof plate as the common site of infection; and pharyngalgia and pain in the neck. On the other hand, clinical manifestations of NPTB with NPC include being old; large, hard and fixed cervical lymph nodes; hearing loss and tinnitus; blood-tinged discharge from the nasopharynx and involvement of the cranial nerves. In that study the diagnosis of NPC was initially missed in 3 of the 6 cases (50\%). Therefore, it is suggested that misdiagnosis of NPTB with NPC could be avoided by improved awareness of NPC and thorough analysis of clinical symptoms of NPTB and NPTB with NPC on top of histopathological examination.

Histopathological examination could be confusing to the pathologist because nasopharyngeal carcinoma may harbor foci of granulomatous reactions. ${ }^{5}$ The granulomatous reactions reflect a favorable local host and cell-mediated immune response. Chen et al. ${ }^{7}$ reported that 7 of 47 cases initially diagnosed as primary NPTB were reexamined and found to have NPC. In routine stains, residual malignant tumor cells could be identified in two cases while in the remaining five cases malignant cells could only be identified after careful examination. However, with the use of immunohistochemical staining for keratin, tumor cells were easily demonstrated engulfing the granulomatous lesions in all seven cases. Several studies from NPC and TB epidemic countries also reported difficulties and confusion in making diagnosis both clinically and histologically. ${ }^{8}$

From the literature review, the previously published studies and case reports were not incidental. They were either suspected TB later diagnosed to have NPC, vice-versa or both coexistent. However, in this case, the patient initially presented with symptoms of chronic tonsillitis with adenoid remnants and the coexistent pathologies were diagnosed incidentally by histopathological examination.

This patient was a therapeutic dilemma initially. After combined discussion among ENT, oncology, infectious disease and pathology teams, the combined treatment was recommended in view of the high prevalence of both diseases in this hospital and the supporting symptoms and investigation results. He recuperated well and currently remains symptom free on regular follow up.

It is very important that in cases like this, the approach should be multidisciplinary with professional inputs from all related fields to achieve the best outcome of treatment for the patient.

\section{ACKNOWLEDGEMENT}

We are grateful to Professor Prepageran Narayanan (FRCS) from the University Malaya of Kuala Lumpur for reviewing this paper.

\section{REFERENCES}

1. Rohwedder JJ. Upper respiratory tract tuberculosis. Sixteen cases in a general hospital. Ann Intern Med. 1974 Jun 1; 80(6):708-713.

2. Rundi C. Understanding tuberculosis: Perspectives and experiences of the people of Sabah, East Malaysia. J Health Popul Nutr. 2010 Apr; 28(2):114-123.

3. World Health Organization. Global Health Atlas. Global Tuberculosis database. Cited 3 May 2010. Available from: http://apps.who.int/globalatlas/dataQuery/default.asp

4. Zalesska-Krecicka M, Krecicki T, Morawska-Kochman M. [Nasopharyngeal carcinoma coexistent with lymph node tuberculosis, diagnostic difficulties - case report]. Otolaryngol Pol, 2005; 59(4):607-9.

5. Wang $\mathrm{H}$, Zhong YQ, Qiu HG. [Diagnosis of nasopharyngeal tuberculosis (NPTB) and NPTB with nasopharyngeal carcinoma.] China Trop Med, 2005,5(2):280-281. Cited 3 May 2013. Available from: http://en.cnki.com.cn/Article_en/CJFDTOTAL-RDYX200502014.htm

6. Chan AB, Ma TK, Yu BK, King AD, Ho FN, Tse GM. Nasopharyngeal granulomatous inflammation and tuberculosis complicating undifferentiated carcinoma. Otolaryngol Head Neck Surg. 2004 Jan; 130(1):125-130.

7. Chen CL, Su IJ, Hsu MM, Hsu HC. Granulomatous nasopharyngeal carcinoma: with emphasis on difficulty in diagnosis and favorable outcome. J Formos Med Assoc. 1991 Apr; 90(4):353-6.

8. Su K, Jiang F, Miao D, He X, Zhang Y. [Nasopharyngeal tuberculosis: an analytical study and report on 12 cases]. Lin Chuang Er Bi Yan Hou Ke Za Zhi. 2002 Aug,16(8):414-5. 